\title{
Ocio Cibernético en Trabajadores de Instituciones Públicas y Privadas de Arequipa
}

\author{
CYBERNETIC LEISURE AMONG WORKERS FROM PUBLIC AND PRIVATE INSTITUTIONS
}

\section{Walter L. Arias Gallegos', Maria Alejandra Masias Salinas²}

1. Psicólogo por la Universidad Nacional de San Agustín y magister en CC. de la Educación con mención en Psicopedagogía Cognitiva. Está diplomado en Gestión de Recursos Humanos y en Gestión de Sistemas Integrados. Es profesor investigador de la Universidad Católica San Pablo donde dirige el Grupo de Investigación Psyché.

2. Estudiante de psicología de la Universidad Católica San Pablo y coordinadora del Grupo de Investigación Psyché.

\section{RESUMEN}

El ocio ha sido una variable poco estudiada en el ámbito laboral y muchas veces mal entendida. En esta investigación se hace una breve descripción de las características de ocio cibernético laboral en trabajadores de empresas públicas y privadas de la ciudad de Arequipa. La muestra está conformada por 152 empleados de empresas privadas y 70 que laboran en instituciones estatales. Como instrumento se usó una ficha de recolección de datos. Los resultados indican que el ocio cibernético es mayor entre los trabajadores de empresas públicas, basándonos en el tiempo invertido con un nivel de significancia de $\mathrm{p}<0,00$; y que las páginas más frecuentadas durante estos periodos de ocio son las redes sociales como facebook, que ocupa el primer lugar de preferencia en ambos tipo de empresas.

(Arias W, Masías M, 2014. Ocio Cibernético en Trabajadores de Instituciones Públicas y Privadas de Arequipa. Cienc Trab. May-Ago; 16 [50]: 88-92).

Palabras claves: OCIO CIBERNÉTICO, TRABAJADOR, TICS.

\section{ABSTRACT}

Leisure has been a variable poorly studied in working contexts, and, manytimes, misunderstood. In this research, a brief description about some features of cybernetic leisure among workers who came from public and private organizations located in Arequipa City was performed. The sample is composed by 152 employees from private companies and 70 workers from public institutions. As instrument, it was used a file card to collect the data. The results indicate that cybernetic leisure is bigger among workers from public institutions, based on spending time with a level of significance of $p<0,00$; and also social networks are the most visited websites, like facebook, which is in first place of preference in both kind of organizations.

Key words: CYBERNETIC LEISURE, WORKER, ICT.

\section{INTRODUCCIÓN}

El ocio ha ocupado una parte esencial en la vida del hombre, de modo que así como Russell ${ }^{1}$ señala que la guerra es lo que ha estimulado el progreso de la humanidad a través de la creación de tecnología bélica, Javier de Zubiría dice que el motor del desarrollo social es la vida recreativa, ya que los múltiples avances científicos han tenido como fin el hacer más simple la vida del hombre. Y ¿para qué?, pues para tener más tiempo libre. Es un hecho histórico que en la antigüedad el ocio era usado para manipular a las masas. Por ejemplo, el rey Atis, del Asia Menor, introdujo el juego de pelota hace 3.000 años para distraer a sus

Correspondencia / Correspondence:

Walter L. Arias Gallegos

Universidad Católica San Pablo.

Urb. Campiña Paisajista s/n Quinta Vivanco, Cercado, Arequipa, Perú.

Tel.: (51-54) 608020

e-mail: walterlizandro@hotmail.com

Recibido: 29 de Mayo 2014 / Aceptado: 14 de Julio 2014 súbditos en épocas de malas cosechas y de hambre. ${ }^{2}$ En Roma, el emperador Cómodo utilizaba las luchas entre gladiadores para mantener contenta a la población, de ahí que se decía que a la población hay que darle "pan y circo". ${ }^{3}$ Sin embargo, el ocio no siempre ha tenido el sentido de recreación y diversión que hoy le damos.

Por ejemplo, el ocio reflexivo era la actividad predilecta para los filósofos griegos y las castas políticas. A través del ocio, los científicos y aristócratas dedicaban horas de horas a pensar, inventar e incubar sus ideas; por tanto, estaba intimamente ligado con los intereses académicos y las actividades intelectuales. ${ }^{4}$ Esta concepción del ocio se mantuvo hasta la edad media donde los teólogos y sacerdotes también dedicaban su tiempo a la actividad contemplativa, además de orar y meditar. Sin embargo, la concepción de ocio cambió radicalmente con la conformación de las pequeñas empresas de clase media durante el periodo del renacimiento. Estas empresas serían "negocios" familiares en las que el trabajo era el valor central, ya que por las necesidades económicas de la época, entre las clases populares no se disponía de tiempo para reflexionar sin fines prácticos. El trabajo en estas empresas negaba espacio y tiempo para el ocio, de ahí su nombre. ${ }^{5}$ Más adelante, con la revolución industrial, esta visión del trabajo y el ocio se iría consolidando con grandes matices de inequidad y 
explotación, que fueron denunciados tanto por filósofos socialistas como liberales. ${ }^{6}$

Debido a esta visión del ocio como antítesis de toda actividad productiva, se ha extendido entre la población el entendimiento de que el ocio solamente se da en términos de tiempo destinado a la recreación. Sin embargo, se define el ocio como "todo aquel periodo durante el cual el individuo se desliga de sus obligaciones profesionales, familiares y sociales y procura la realización voluntaria de ocupaciones consideradas a priori satisfactorias y como un fin en sí mismo".

Es decir, que el ocio emana de los intereses de la persona, y aunque sí contempla las actividades de recreación, abarca también diferentes pasatiempos. Una caracterización común del ocio es dividirlo entre ocio activo y ocio pasivo. En el ocio activo las actividades que se realizan son productivas, es decir que la persona hace algo, crea algo, o se ocupa en una actividad demandante que es placentera. Hacer ejercicio, leer, escribir, armar modelos a escala, son ejemplos de ocio activo. En el ocio pasivo en cambio, la persona no hace nada productivo, aunque la actividad como tal genera disfrute (i.e. dormir, ver televisión, etc.). Con respecto al ocio pasivo, se ha visto que reduce la calidad de vida y que se asocia con estados emocionales negativos. Las personas solitarias por ejemplo, llenan su tiempo libre con ocio pasivo. Además, ya en 1958 el Grupo para el Progreso de la Psiquiatría señaló que el "ocio es peligroso para el pueblo norteamericano", porque se había notado que una enorme cantidad de hechos trágicos ocurren durante los momentos de ocio. De otro lado, Sandor Ferenczi (1873-1933), conocido psicoanalista, hablaba de la "neurosis dominical" para referirse a un estado emocional negativo que vivían las personas el día domingo, porque no encontraban nada que hacer con su tiempo libre, lo cual las deprimía y las orillaba a tener pensamientos negativos y hasta optar por el suicidio $^{2}$. En ese sentido, Erich Fromm (1900-1980) ${ }^{8}$ había señalado que una persona que no es capaz de pasar tiempo sola sin hacer nada no está bien consigo misma, no se soporta, ni se acepta. Romano Guardini señala, por eso, que es fundamental la aceptación del sí mismo para poder vivir de manera satisfactoria. ${ }^{9}$

De todo esto podemos concluir que no todas las actividades de tiempo libre son iguales. Mientras que el ocio activo conlleva a experimentar estados de sosiego y fluidez, el ocio pasivo nos desgasta y nos deprime. Las pruebas señalan incluso que el tiempo libre es más difícil de disfrutar que el trabajo, pero para las personas flow el trabajo es tan placentero como el ocio, ya que se dedican a actividades que les conducen hacia su propia autorrealización. ${ }^{2}$

Dada la masificación de las TICs, el ocio está dedicado en buena medida a la recreación a través de la tecnología. En ese sentido, quizá uno de los medios de comunicación que pronto comenzó a acaparar el ocio de los jóvenes y de las familias fue la televisión. De acuerdo con un estudio efectuado por Aurum consultores en la ciudad de Arequipa el 2012, se encontró que el 90\% de varones ve televisión, pero este porcentaje es menor en sectores $A B$, mientras que para el caso de las mujeres, solo el 9\% ve televisión en los sectores $\mathrm{C}$ y D. ${ }^{10}$ Esto sugiere que en los sectores populares una gran mayoría de hombres utiliza la televisión como medio de distracción, pero las mujeres de su misma condición social se dedican al trabajo doméstico (85\%). En otro estudio, entre las actividades que realizan como familia en su tiempo libre, hemos reportado que en Arequipa las actividades preferidas son dormir, leer, salir a pasear o pasar tiempo con los amigos. ${ }^{11}$
En una investigación realizada en España, se encontró que el 43\% de su tiempo libre los jóvenes universitarios lo usan para ir a bares y a beber. Se identificaron también algunos factores que determinan el tipo de ocio, como son el momento de ocio (horario diurno-nocturno), el coste del ocio (barato-caro), tono del ocio (tranquilo-bullicioso) y la variabilidad de las actividades (variableestable). ${ }^{7}$ En ese sentido, puede decirse que el ocio está determinado por el nivel socioeconómico, ya que el poder adquisitivo juega un papel muy importante en la elección de las actividades de ocio. El ocio turístico es, por ejemplo, una de las actividades preferidas de las personas de clase media alta y clase alta. ${ }^{12} \mathrm{La}$ cultura nacional, o la idiosincrasia de un pueblo, también puede ser determinante en el ocio. Se sabe que en los países individualistas y/o capitalistas el ocio laboral es mayor que en los países colectivistas o socialistas. ${ }^{13}$

Por otro lado, hoy en día el uso de la internet y de otras tecnologías móviles ha hecho que diversas actividades humanas tengan otro significado. ${ }^{14}$ Una de estas actividades es el trabajo. En Chile, por ejemplo, el 25\% de las empresas usa el teletrabajo como modalidad de producción. ${ }^{15}$ En el Perú, aparecen con internet nuevas formas de interacción que han impactado en los patrones de uso del tiempo libre -y sobre todo en los jóvenes-, lo cual ha generado diversas ventajas, pero también inconvenientes. Los estudios de Roselli señalan en ese sentido que, aunque la interacción social es mayor en la comunicación personal, el trabajo es mejor por el chat. ${ }^{16}$ Pero en un sentido negativo, es necesario controlar la información que se encuentra en internet porque hay páginas que se especializan en la asistencia al suicidio, orientan el desarrollo de un trastorno alimentario, promueven la pornografía o la pederastia. ${ }^{14}$

Ahora bien, el ocio como tal cobra dimensiones particulares cuando se habla de ocio laboral, es decir, que tiene lugar en el trabajo. En términos tradicionales se puede decir que el ocio se contrapone al trabajo y, de hecho, históricamente los trabajadores han luchado por su derecho al tiempo libre. ${ }^{17}$ Un trabajador ocioso es mal visto porque se desvía de sus actividades laborales $\mathrm{y}$, por tanto, produce menos, pero sorprendería saber que en un experimento de Frederick W. Taylor (1856-1915), éste logró aumentar la producción diaria de $12,5 \mathrm{TN}$ a $47 \mathrm{TN}$ dejando que los obreros descansen la cuarta parte del tiempo de trabajo. ${ }^{18}$

Por otro lado, así como el ocio se diferencia del ocio laboral, el ocio cibernético en el trabajo tiene también sus propias consideraciones, pues básicamente implica el uso indebido de las TIC, sobre todo internet, en horas de trabajo. Así pues, si bien internet se puede usar en ratos libres -el 58\% de personas considera que internet llena un espacio reservado para el ocio ${ }^{14}$ - no debe chocar con las horas de trabajo ni transgredir las normas de conducta. Pero dado que el ocio cibernético se ejerce a través de las tecnologías de la información y comunicación, su control es sumamente complejo y difícil. Algunas medidas que se han adoptado para limitar el ocio cibernético en el trabajo pasan por impedir la instalación de juegos y software no relacionados con las actividades laborales. Por otra parte, dado que el ocio cibernético gira en torno a internet, la restricción de las páginas web es quizá la medida más usada.

Quizá el uso tan extendido del internet se deba a que es usado como un agente socializador, lo cual permite satisfacer las necesidades de pertenencia de grupo de las personas. ${ }^{19} \mathrm{El}$ chat es un recurso tecnológico que favorece la socialización, ya que en el chat se emiten mensajes que reproducen la realidad social, a la 
vez que se la transforma. Asimismo, para el uso del chat, y de las TICs en general, no hay distinciones de sexo, edad o condición socioeconómica. Aunque se ha reportado que los varones prefieren usar los juegos en línea y navegar por internet, mientras que las mujeres prefieren chatear y usar las redes sociales. También se ha señalado que los adultos mayores usan menos las TICS y que el nivel de ingresos y la ubicación geográfica pueden limitar su acceso. ${ }^{20}$

Es importante tener en cuenta que la conectividad no se reduce solo a lo tecnológico sino que abarca también lo social. Por esta razón, los teléfonos celulares pasan a ser parte de la vida diaria de las personas. Su uso masivo ha influido en su renovación tecnológica de manera muy acelerada, de tal manera que ahora es posible acceder a un sinfín de recursos propios de internet a través de los celulares. De esta forma el celular puede usarse como instrumento de comunicación personal, como recurso para una interacción constante, como sistema de ayuda, como recurso lúdico o de entretenimiento, como medio testimonial, como signo de estatus, como elemento de control, instrumento de negocios y de movilización social, etc. ${ }^{21}$

El celular es, también, más usado por los jóvenes porque los mensajes de texto facilitan el uso de múltiples canales de comunicación en simultáneo y se valida la comunicación oral y escrita. Sin embargo, ellos lo usan mayoritariamente con fines de estudio y juego. ${ }^{22}$ Una investigación realizada en Arequipa se reportó que un $5,9 \%$ de la población universitaria era adicta al uso del celular. ${ }^{23}$ En ese sentido, el uso de las TICs no tiene nada de malo per se, pero cuando la tecnología es usada de modos o en lugares indebidos, sobrevienen los problemas. En un estudio hecho en México se encontró que el 34\% de los cibernautas mexicanos accede a internet en el trabajo, 13\% lo hace en cibercafés y un $8 \%$ en la escuela; y que la mayoría chatea sobre temas relativos al sexo. ${ }^{24}$ Según la Rosa ${ }^{19}$, las redes sociales son el entorno en línea al que más acceden las personas, y que los sitios más visitados de internet son Facebook, Youtube, Blogger, Twitter, Wordpress.com, Linkedin, My space, etc. De acuerdo con Escalante, la preferencia por el uso de las redes sociales se explica porque refuerza los vínculos y permite desarrollar una identidad "mejorada", lo cual es estimulante y tiene un impacto positivo en la autoestima de las personas. $^{25}$

El uso indebido de las TICs ha generado un intenso debate, ya que en base al abuso que se hace de ellas o de la dependencia que puede generar en los cibernautas, se ha propuesto aplicar los criterios de adicción a las sustancias psicoactivas al uso de la internet. ${ }^{26}$ Puede decirse que las adicciones a las TICs comienza con las investigaciones sobre las adicciones a los videojuegos ${ }^{27}$, que si bien se mantienen ${ }^{28}$, han dado paso a los estudios en adicciones al celular y a la internet. ${ }^{23,29} \mathrm{Al}$ respecto, debe tomarse en cuenta que si bien los jóvenes están más propensos a estos desórdenes $^{30}$, los problemas conductuales que acarrea son de consideración. ${ }^{31}$ En tal sentido, cuando se presentan adicciones al internet o problemas no clínicos relacionados con su uso, la esfera laboral suele verse también comprometida.

El presente trabajo no pretende ahondar el tema de la adicción a internet, sino que solamente busca analizar la incidencia y las preferencias más frecuentes en el uso de internet dentro del horario de trabajo; por tanto, se enmarca en el campo del ocio cibernético. De manera adicional, se hace una comparación del ocio cibernético en empresas públicas y privadas, bajo la premisa de que éste es mayor en las empresas públicas porque existen menos mecanismos de control y mayor burocracia. Este trabajo, además, forma parte de una investigación más extensa sobre el uso de las TICs en el trabajo.

\section{MÉTODO}

\section{Muestra}

La muestra está conformada por 152 trabajadores de ocho empresas privadas y 70 trabajadores de cinco empresas públicas en la ciudad de Arequipa, que se ubica al sur del Perú. De la empresa privada, el 55,9\% está formado por varones y el 44,1\% por mujeres, con una media de edad de 25.51 años con una desviación estándar de \pm 3,906. De la empresa pública, el 55,7\% es varón y, el 44,3\%, mujer, con una edad media de 35,85 años con una desviación estándar de $\pm 10,107$. Se realizó un muestreo intencional para seleccionar las empresas y se utilizó la técnica de muestreo de sujetos voluntarios. Se trató, por tanto, de una muestra no probabilística.

\section{Instrumentos}

Como instrumento se usó una ficha de recolección de datos, donde se registran la información general de la empresa (nombre, rubro, tipo) y del trabajador (sexo, edad, ocupación, etc.). Además se incluyen tres preguntas para el trabajador sobre las horas que pasa navegando en internet, los sitios web que visita y las redes sociales a las que accede.

\section{Procedimiento}

Los trabajadores fueron encuestados vía e-mail con una ficha de recolección de datos, en sus lugares de trabajo, con la autorización de sus jefes y las autoridades superiores. El tiempo aproximado de participación por persona fue de dos minutos. Los datos se recogieron de lunes a viernes en horario de trabajo durante el segundo semestre del año 2012.

\section{RESULTADOS}

Con respecto al tiempo que le dedican en el trabajo al ocio cibernético encontramos que los empleados del sector privado usan entre 1 y 4 horas al día, con una media de 1.685 horas y una desviación estándar de $\pm 0,480$; mientras que en las instituciones públicas se dedica mínimo una hora y máximo seis horas al ocio cibernético, con una media de 3.786 horas por dia y una desviación estándar de $\pm 2,152$.

Asimismo, puede afirmarse que la diferencia entre estos valores es significativa, ya que se obtuvo un valor t de 0,473 y una t crítica de 0,178 , lo cual indica que las diferencias no se deben al azar, con un nivel de significancia de $\mathrm{p}<0,00$. Estos datos corroboran nuestra hipótesis, así que se rechaza la hipótesis nula y se acepta la hipótesis de investigación.

Por otro lado, acerca de las páginas web más buscadas se encontró que el 17\% de trabajadores de las instituciones públicas visita lugares de carácter sexual, 57,1\% navega en las redes sociales y $18,6 \%$ juega en internet, mientras que solo el 7,1\% busca información. En la empresa privada el 12,9\% busca web sites sobre sexo, el 53,4\%, usa las redes sociales -de este grupo, 28,6\% es mujer y $25,7 \%$ es varón-, 5,7\% juega con la computadora y 27,1\% busca información (ver Figura 1). 
Figura 1.

Porcentajes de páginas web más buscadas.

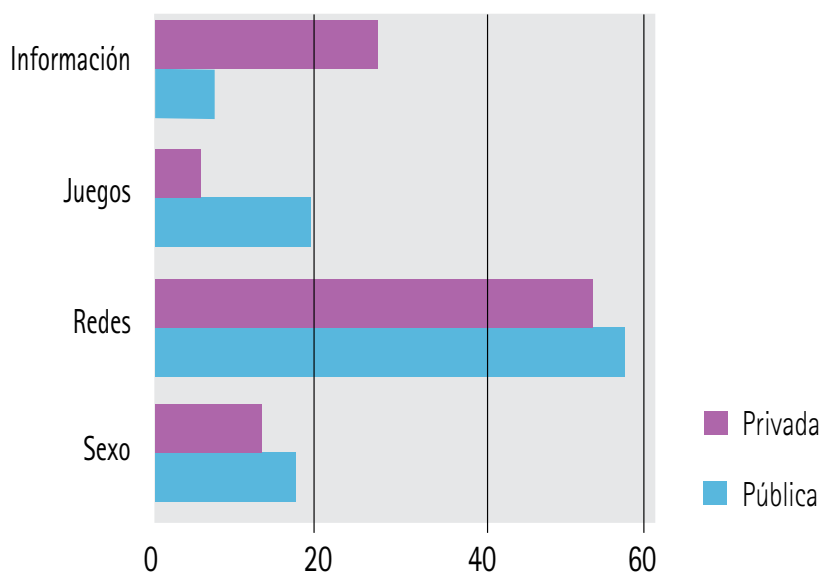

Figura 2.

Porcentajes de redes sociales.

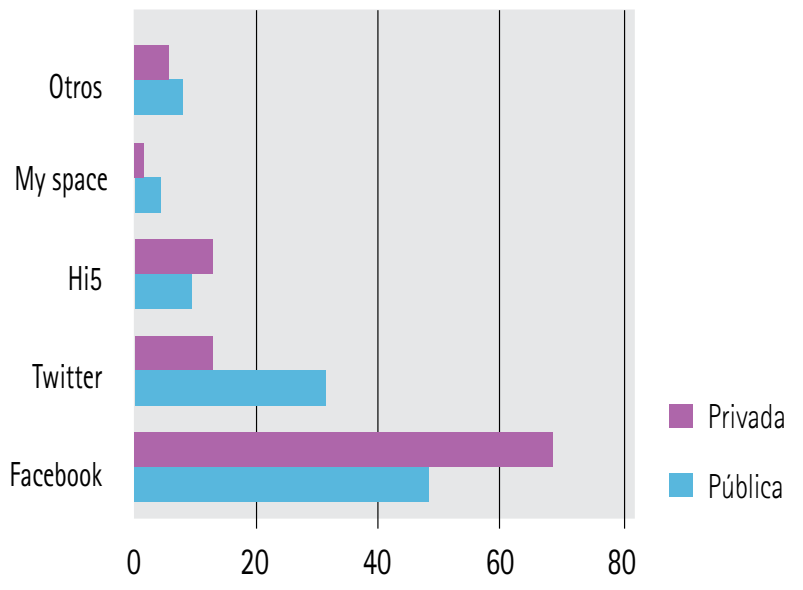

Finalmente, en las empresas privadas, el 68,4\% de los trabajadores usa preferentemente el facebook, 12,5\% usa twitter, el 12,5\% usa hi5, 5,3\% usa My space y solo el 1,3\% usa otras redes sociales. En las instituciones públicas, por otro lado, el $48 \%$ de los empleados tiene facebook, 30,9\% usa twitter, 9,2\% usa hi5, 3,9\% usa My space y 7,9\% usa otra red social (ver Figura 2).

Estos datos, vistos en su conjunto, nos indican que el ocio cibernético no solo es mayor en las instituciones públicas sino que, además, el tipo de actividades que se realizan se encuentran diferenciadas aunque comparten también ciertas particularidades.

\section{DISCUSIÓN}

El ocio es una de las actividades a las que el ser humano dedica más tiempo. Muchas y -sobre todo-, diversas actividades socioeconómicas giran en torno al ocio: el 7\% de la energía no renovable que usamos se va en el ocio. ${ }^{2}$ De modo que el ocio cumple una función revitalizante, de descanso y homeostasis, cuando se asocia a actividades pasivas que satisfacen necesidades básicas, y de autorrealización cuando se realiza como una actividad que permite satisfacer necesidades espirituales.
Nuestro estudio nos permite señalar que el ocio cibernético es mayor entre trabajadores de instituciones públicas que entre las personas que trabajan en empresas privadas, basándonos en el tiempo que dedican al ocio cibernético en el trabajo. Este dato es congruente con el hecho de que las instituciones públicas registran altos niveles de burocracia. En el año 1987, De Soto, Ghersi y Ghibellini ${ }^{32}$ realizaron un estudio clásico que dejó traslucir cómo los altos índices de burocracia tienen un impacto negativo en el proceso de formalización de las microempresas peruanas. De acuerdo con este estudio, obtener las licencias de funcionamiento de una empresa costó más de mil dólares y tomó casi un año de trámites y gestiones. Hoy en día, la burocracia de las instituciones estatales peruanas ha disminuido, pero todavía genera dolores de cabeza. Posiblemente, el hecho de navegar en internet en lugar de dedicarse a las labores cotidianas sea un factor de las dilaciones de los procesos burocráticos en muchas empresas del pais, sobre todo las dedicadas a la jurisprudencia. Por otro lado, las actividades de ocio cibernético en el trabajo presentan matices muy interesantes en función de las páginas visitadas, ya que si bien en ambos tipos de empresas sobresale el uso a las redes sociales, los juegos y la información relativa al sexo alcanzan valores inversos: mientras en las empresas privadas la opción más marcada después de las redes sociales es el sexo, en las empresas públicas son los juegos. Quizá esto tenga relación con la edad de los trabajadores, ya que los empleados de las empresas privadas son menores en casi diez años que los trabajadores de las empresas públicas, tomando en consideración las medias de las edades. Por un lado, los videojuegos son usados de forma excesiva o dependiente por personas cuyo rango de edad va desde los 12 hasta los 30 años ${ }^{28}$, si consideramos que los videojuegos han ingresado a nuestro medio en la década de los 80, y la internet en los 90', fácilmente podemos inferir que los trabajadores de hoy en día han vivido en contacto con estas tecnologías lúdicas, por ello es factible que algunos de sus hábitos se relacionen con los juegos en red. Por otro lado, la preferencia por páginas de contenido sexual, sean softcore o hardcore, han sido asociadas con la personalidad, de modo que quienes son más retraídos o tienen mayores dificultades para relacionarse con los demás suelen consumir este tipo de información y buscan hacer contacto con otros por internet, por eso es que también suelen hacer uso frecuente de las redes sociales ${ }^{26}$. Actualmente, estamos realizando un estudio sobre la personalidad y el uso de la computadora en jóvenes universitarios, bajo la premisa de que quienes son más introvertidos son los que tienen un uso mayor.

En ese sentido, son precisamente los jóvenes quienes recurren en mayor medida a la internet y al uso de las TICs con fines lúdicos ${ }^{29,30}$, siendo uno de sus recursos favoritos el acceso a las redes sociales. Nuestro estudio ha arrojado que facebook es la red social más admitida por los trabajadores de empresas tanto públicas como privadas, y le siguen twitter, hi5 y My space. Sin embargo, otros estudios reportan que, a nivel global, el 74\% de jóvenes tiene twitter, el 43\% tiene facebook y el 38\% tiene My space. $^{32}$

Es interesante notar cómo los jóvenes han venido cambiando sus hábitos de ocio, ya que las actividades deportivas y de ejercicio físico han quedado rezagadas por el uso de las TICs. ${ }^{33}$ Hoy en dia, conectarse a internet es entre los jóvenes la primera actividad de ocio, por encima de salir a beber, ir a fiestas, ver televisión, salir de compras, hacer deporte o jugar videojuegos. ${ }^{34}$ 
Finalmente, debemos considerar algunos factores que definitivamente limitan el alcance de nuestros resultados. Primero, el tipo de muestreo no nos permite analizar con detalle los datos en trabajadores de mayor edad, ya que, como se usó un muestreo de sujetos voluntarios, tuvimos que atenernos a quienes desearon colaborar libremente con la investigación, que fueron jóvenes en su mayoría. Es importante, empero, abarcar población de mayor edad y de forma sistemática y aleatoria, porque ellos son migrantes digitales, mientras que los jóvenes son nativos digitales. ${ }^{20}$ Este hecho es significativo, para poder comprender la conducta de las personas frente a la introducción de la tecnología en el trabajo, y hacer generalizaciones válidas. Los datos de nuestro estudio no son representativos de las empresas públicas o privadas de Arequipa, pero nos muestran datos que ameritan ser objeto de estudio más amplio, y hacia esa dirección nos estamos moviendo.

En segundo lugar, el instrumento usado es una ficha de registro que no permite medir de manera fehaciente las variables de estudio. En trabajos más recientes estamos midiendo diversos valores de variables de estudio como personalidad, adicción a internet, tecnoestrés, etc. que seguramente nos darán mayores luces sobre la compleja panorámica que se ha generado con el uso de las TICs. Solo podemos concluir que el ocio cibernético es una variable que muy pocas veces se toma en cuenta, pero que en realidad está siempre presente en el trabajo; antes de abordarla, tenemos que comprenderla. Este pequeño estudio espera haber podido motivar el interés sobre un tema que es ignorado en el contexto laboral.

\section{REFERENCIAS}

1. Russell B. El impacto de la ciencia en la sociedad. Madrid: Aguilar, S. A. Ediciones; 1957.

2. Csikszenmihalyi M. Aprender a fluir. Barcelona: Kairós; 2012.

3. Leahey T. Historia de la psicología. México: Pearson Educación; 2006.

4. Pieper J. El ocio y la vida intelectual. Madrid: Rialp; 1998.

5. Hubeñak F. Historia integral de occidente desde una perspectiva cristiana. Buenos Aires: EDUCA; 2009.

6. Arias WL. Revisión histórica de la salud ocupacional y la seguridad industrial. Rev Cub de Salud Trab. 2012;13(3): 45-52.

7. Garcia-Cueto E, Cerro V. Características individuales y tipo de ocio. Psicothema. 1990; 2(2):173-177.

8. Fromm E. El arte de amar. Buenos Aires: Paidós; 1964.

9. Guardini R. La aceptación de sí mismo. Buenos Aires: Lumen; 2011.

10. Aurum. Los arequipeños: Actividades y expectativas. Arequipa Empresarial. 2012;17: 30-31.

11. Castro $R$, Arias, WL, Domínguez $S$, Masias MA, Salas $X$, Canales F, Flores A. Integración familiar y variables socioeconómicas en Arequipa metropolitana. Rev Investig (Arequipa). 2013; 4:35-65.

12. Alvarez A. El ocio turistico en las sociedades industriales avanzadas. Barcelona: Bosch Casa Editorial S. A.; 1994.

13. Robbins $S$, Judge T. Comportamiento Organizacional. México: Pearson Educación; 2009.

14. Trujano P, Dorantes J, Tovilla V. Violencia en internet: Nuevas victimas, nuevos retos. Liberabit. 2009;15(1):7-19.

15. Gareca $M$, Verdugo $R$, Briones J $L$, Vera A. Salud ocupacional y teletrabajo. Ciencia \&t Salud. 2007; 9(25): 85-88.

16. Roselli N. Proceso de construcción colaborativa a través del chat según el tipo de tarea. Revista de Psicología. 2011; 29(1):3-36.

17. Miranda G. El tiempo libre y ocio reivindicado por los trabajadores. Pasos. 2006; 4(3):301-326.

18. Smith CS, Wakeley H. Psicología de la conducta industrial. México: McGrawHill; 1977.

19. La Rosa A. Redes sociales en la web, comunicación y comportamiento social. Av psicol. 2010; 18(1): 85-95.

20. Barrantes R. Análisis de la demanda por TICs: ¿Qué es y cómo medir la pobreza digital? En: Barrantes R. Pobreza Digital: Las perspectivas de América Latina y
El Caribe. Lima: DIRSI; 2007.

21. La Rosa A. Teléfonos móviles, comunicación e interacción. Av psicol. 2012; 20(1):69-79.

22. Coppari N, Angulo N, Constantini GM, Ferreira JJ, Martínez MR, Peris MS, Vásquez MJ. Uso de nuevas tecnologias y su relación con tecnoestrés en adolescentes escolarizados. Rev Per Psi y Trab Soc. 2013; 2(2):97-110.

23. Morales LF. Adicción al teléfono móvil o celular. Rev psicol Arequipa. 2012; 2(1):94-102.

24. Rocha MA. El lenguaje de los jóvenes en el chat. Estudios sobre las culturas contemporáneas. 2004;10(19):109-140.

25. Escalante E, Agrelo A. Explorando experiencias en el facebook: Identidad, vínculos sociales, privacidad. Rev Per Psic Trab Soc. 2012;1(1):69-88.

26. Navarro AA, Rueda GE. Adicción a internet: Revisión crítica de la literatura. Rev Colom Psiq. 2007; 36(4):691-700.

27. Tejeiro R. Adicción a los videojuegos. Una revisión. Adicciones. 2001;13 (4):407-413.

28. Vallejos $\mathrm{M}$, Capa W. Video juegos: Adicción y factores predictores. Av Psicol. 2010; 18(1):103-110.

29. Sánchez-Carbonell $X$, Beranuy $M$, Castellana $M$, Chamorro $A$, Oberst U. La adicción a internet y al móvil: ¿moda o trastorno? Adicciones. 2008; 20(2):149-160.

30. Castellana M, Sánchez-Carbonell X, Graner C, Beranuy M. El adolescente ante las tecnología de la información y la comunicación: internet, móvil y videojuegos. Papeles del Psicólogo. 2007; 28(3):196-204.

31. Blanco MG, Anglada MV, Fernández MJ, Arbonés MM. Problemas conductuales relacionados con el uso de internet: Un estudio exploratorio. Anales de Psicología. 2002; 18(2):273-292.

32. De Soto H, Ghersi E, Ghibellini M. El otro sendero. Lima: Instituto Libertad y Democracia; 1987

33. Aguillo IF, Ortega JL, Granadino B. Contenidos del buscador Google. Distribución por países, dominios e idiomas. El profesional de la información. 2006; 15(5): 384-389.

34. Ramos R, Valdemoros MA, Sanz E, Ponce de León A. La influencia de los profesores sobre el ocio físico deportivo de los jóvenes: percepción de los agentes educativos más cercanos a ellos. Profesorado. 2007; $11(2): 1-18$. 